\title{
Transport Properties of Conductive Polyaniline Nanocomposites Based on Carbon Nanotubes
}

\author{
S. B. Kondawar ${ }^{*}$, M. D. Deshpande, S. P. Agrawal \\ Department of Physics, RTM Nagpur University, Nagpur, 440033, India \\ sbkondawar@yahoo.co.in,mdd.milind@gmail.com, a_shikhaagrawal@yahoo.com
}

\begin{abstract}
Intrinsically conducting polymers have been studied extensively due to their intriguing electronic and redox properties and numerous potential applications. To improve and extend their functions, the fabrication of multifunctional conducting polymer nanocomposites has attracted a great deal of attention with the advent of nanoscale dimension. In this paper we report the comparative study of nanocomposite synthesized by an in-situ oxidative polymerization of aniline monomer in the presence of functionalized multiwall carbon nanotubes (MWCNT) with that of pure polyaniline (PANI). Fourier transform infrared spectroscopy (FTIR), scanning electron microscopy (SEM) and x-ray diffraction (XRD) are employed to characterize the pure PANI and the PANI-CNT nanocomposite. XRD and SEM reveal the homogeneous coating of PANI onto the CNT indicating that carbon nanotubes were well dispersed in polymer matrix. The interaction between the quinoid ring of PANI and the MWCNT causes PANI chains to be adsorbed at the surface of MWCNT, thus forming a tubular core surrounding the MWCNT was confirmed from FTIR. Nanocomposite shows high electrical conductivity compared to pure PANI. The enhancement in conductivity of the nanocomposite is due to the charge transfer effect from the quinoid rings of the PANI to the MWCNT. The effect of MWCNT on the transport properties of PANI in the form of the transport parameters such as charge localization length, most probable hopping distance and charge hopping energy in the temperature range $300-430 \mathrm{~K}$ was also studied.
\end{abstract}

Keywords Carbon Nanotubes, Conducting Polymer, Polyaniline, Nanocomposites

\section{Introduction}

Organic polymers capable of conducting electricity due to partial oxidation or reduction (i.e., doping), are prospective classes of advanced materials. They possess an extended $\pi$-conjugation along the polymer backbone and exhibit semiconducting behaviour. During the last few decades, electrical transport in such conducting polymers has been thoroughly studied[1-4]. Organic conducting polymers exhibit the electrical and optical properties of the metals and retain the attractive mechanical properties of polymers leading to wide range of technological applications. After the report of preparation of carbon nanotubes and polymer composites by Ajayan et al[5], there have been efforts to combine carbon nanotubes and polymers to produce functional composite materials with desirable electrical and mechanical properties[6-8]. Hence some properties of polymer have been exploited by incorporating the nanomaterials into the polymer matrix. Carbon nanotubes have attracted considerable attention due to their potential application in electronic devices. They have unique structural,

* Corresponding author:

sbkondawar@yahoo.co.in (S. B. Kondawar)

Published online at http://journal.sapub.org/cmaterials

Copyright (C) 2012 Scientific \& Academic Publishing. All Rights Reserved mechanical, electronic, and thermal properties and are attractive building blocks for the development of novel polymer-nanocomposite materials with enhanced functionality, especially if it comes to enhanced conductivity, thermal stability, and reinforcement properties[9-12]. Sambhu Badra et al[13] reported the superiority of polyaniline over all other conducting polymers. Polyaniline (PANI) is taken as matrix material for our work, because not only it is highly stable in air and in some solvents, but also exhibits dramatic changes in its electronic structure and physical properties Polymerization in the presence of carbon nanotubes leads to a more planar conformation of PANI along multiwall carbon nanotubes (MWCNT). Because of the formation of conducting polymer-CNT networks, these materials are of interest for electronic applications including photovoltaic cells, organic light emitting diodes, electromagnetic shielding, electro static dissipation, antennas, and batteries[14-16]. There is a lot of work reported on the preparation of polyaniline-CNT composites to improve the electrical conductivity of the host polymer, but no one reported the change in transport parameters of the composites. Yi Zhou et al[17] reported the strong interaction in conjugated systems greatly improves the charge-transfer reaction between polyaniline and the carbon nanotube prepared by an in-situ polymerization of aniline monomers using multi-wal 
led carbon nanotubes with minimized defects as templates. Polyaniline coated multiwalled carbon nanotubes as additives has been used for enhancing the electrical conductivity of nylone 6 composite was studied by R. R. Vankayala et al[18]. Qi Jianga et al reported the preparation of carbon nanotube/polyaniline nanofiber by electrospinning[19]. Modification of glassy carbon electrode with polyaniline/multi-walled carbon nanotubes composite prepared by Liang Ding et al[20]. Subhodh Srivastva et al[21] reported multiwall carbon nanotube (MWNT) doped polyaniline (PANI) composite thin films for hydrogen gas sensing applications. Due to van-der Waals forces, tight bonding of CNT limits their applications. In most of the work reported, MWCNTs have not functionalized before incorporated into PANI matrix. Therefore in our study MWCNT surface is functionally modified by ultrasonication using $\mathrm{H}_{2} \mathrm{SO}_{4}$ and $\mathrm{HNO}_{3}$ to provide specificity for improved interaction between CNT and polymer matrix which enhances the processability and properties of composites. This work describes the synthesis and characterization of protonic acid doped PANI functionalized MWCNT fabricated by an in-situ chemical oxidative polymerization method. First time we present the detailed electrical transport properties[22] of PANI/MWCNT composites, in the form of the transport parameters such as de electrical conductivity $(\sigma)$, charge localization length $\left(\alpha^{-1}\right)$, most probable hopping distance $(R)$ and charge hopping energy $(w)$ in the temperature range $300-430 \mathrm{~K}$.

\section{Experimental}

\subsection{Materials and Methods}

Aniline (99\%) and ammonium persulphate (APS) (99\%) were purchased from Merck. Aniline was distilled before use for polymerization. High purity MWCNT of diameter 30-40 nm was made available from NPL, New Delhi, India. Other supplement chemicals were of AR grade and used as received.

$\mathrm{X}$-ray diffraction (XRD) analysis was carried out on Philips PW1710 automatic X-ray diffractometer with $\mathrm{Cu}-\mathrm{K}_{\alpha}$ radiation $(\lambda=1.5404 \AA)$, with a scanning speed of $10 \mathrm{~min}^{-1}$. FTIR spectra were performed on Shimatzu FTIR-8101A Spectrophotometer in the wavelength range of 400-4000 $\mathrm{cm}^{-1}$. SEM images were taken on JEOL JSM-6360 analytical scanning electron microscope. The electrical conductivity of the compressed pellets of PANI-MWCNT nanocomposites was determined by four probe resistivity technique. The pellets were prepared with the help of hydraulic press (Kimaya Engineers, India) by applying a pressure of 5000 $\mathrm{kg} / \mathrm{cm}^{2}$.

\subsection{Functionalization of MWCNT}

The solution of $6 \mathrm{M} \mathrm{H}_{2} \mathrm{SO}_{4}$ and $6 \mathrm{M} \mathrm{HNO}_{3}$ in 3:1 ratio was stirred for 10 minute. MWCNT was added to it and then solution was sonicated for 4 hours at $50^{\circ} \mathrm{C}$. After centrifugation MWCNT was filtered, washed and dried to get func- tionalized MWCNT.

\subsection{Synthesis of PANI-MWCNT Nanocomposite}

The synthesis of PANI-MWCNT nanocomposite was performed using in-situ oxidative polymerisation by dispersing $100 \mathrm{mg}$ of functionalized MWCNT in $1 \mathrm{M} \mathrm{HCl}$ in order to have a monomer/MWCNT weight ratio of 100/1, and using a monomer/oxidant molar ratio of $1 / 1$ as described by Zein et al[23]. The pure conducting polymer (PANI) was synthesised by following the same steps carried out for the nanocomposite without MWCNT. The final products obtained from the syntheses were the emeraldine salt forms of PANI-MWCNT nanocomposite and PANI pure polymer.

\section{Results and Discussion}

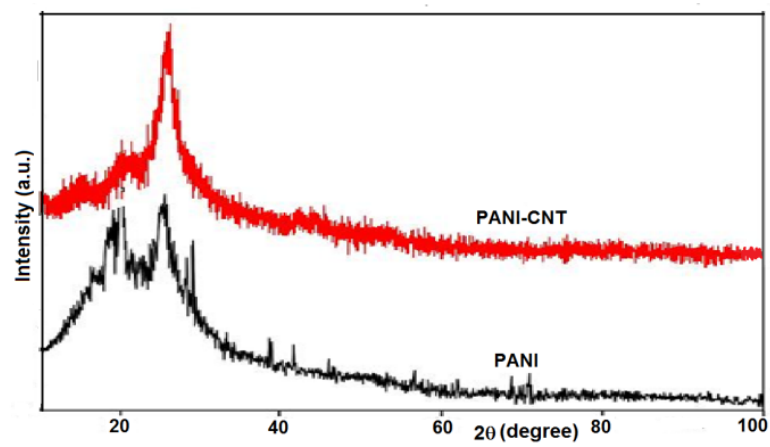

Figure 1. XRD pattern of PANI-CNT and pure PANI

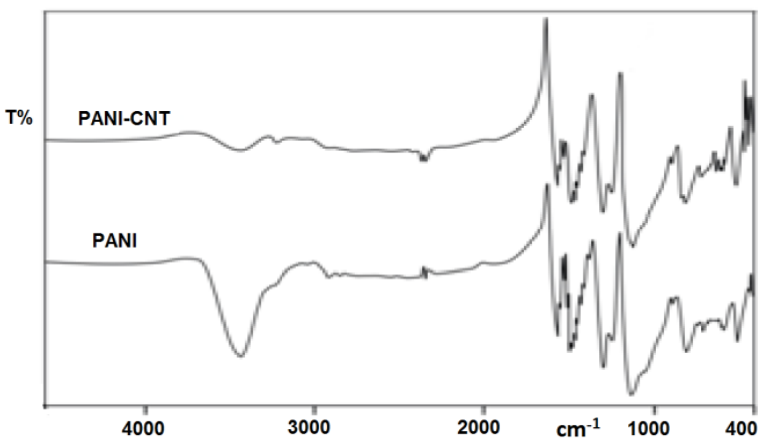

Figure 2. FTIR spectra of PANI-CNT and pure PANI

The XRD pattern of PANI-CNT and pure PANI are shown in figure 1. For PANI, the characteristic peaks appeared at 15.3, 20.4 and 26.28, corresponding to (011), (020) and (200) crystal planes of PANI[20]. When carbon nanotubes were incorporated into the PANI matrix, the sharp and strong diffraction peak of CNT at (26.28) was observed as overlap with the peak of PANI which results in the broad and intense peak in the composite. The data indicates that no additional crystalline order has been introduced into the composite. Compared with functionalized CNT, the obvious characteristic peaks in PANI/CNT can be ascribed to the formation of crystal appearing on the outer layers of nanotubes. This result shows that the homogeneous coating of PANI onto the CNTs indicating that CNTs were well dispersed in polymer matrix as seen from SEM also. 

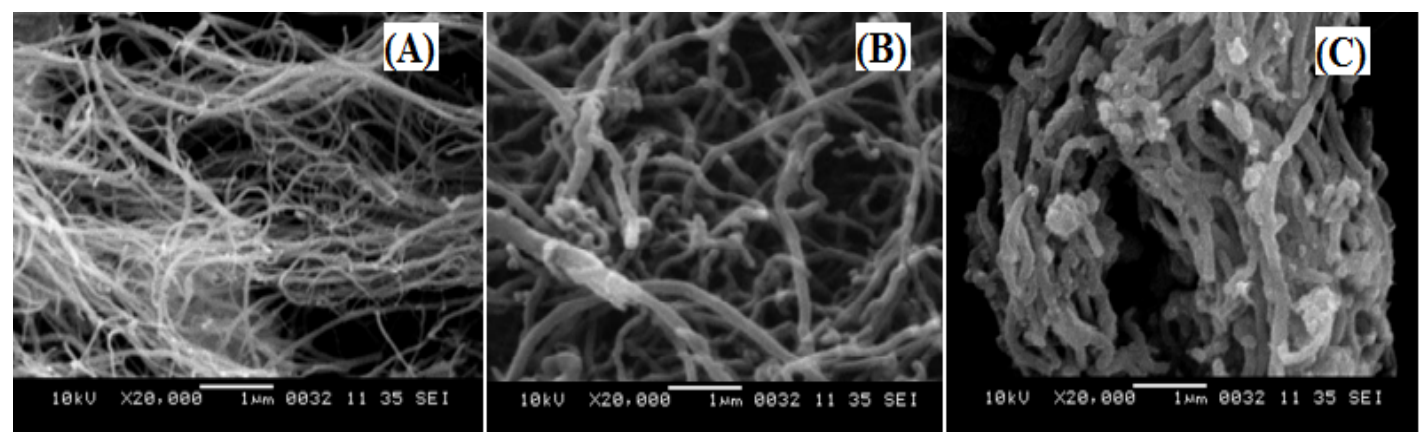

Figure 3. SEM of MWCNT (A), functionalized MWCNT (B), and PANI-CNT composite (C)

The FTIR spectra of PANI-CNT and pure PANI are shown in figure 2 . The peak at $835 \mathrm{~cm}^{-1}$ is attributed to the $\mathrm{N}-\mathrm{H}$ out-of-plane bending absorption. Presence of benzoid band around $1470 \mathrm{~cm}^{-1}$ and quinoid vibration around 1580 $\mathrm{cm}^{-1}$ indicate the emeraldine salt form of PANI and PANI-CNT composite. The band at $3400 \mathrm{~cm}^{-1}$ indicates the stretching of N-H band of aromatic ring in PANI and composite. The peak at around $1590 \mathrm{~cm}^{-1}$ corresponds to the IR active phonon mode of the CNT and the peak at around 1730 $\mathrm{cm}^{-1}$ corresponds to the stretching mode of the carboxylic acid groups, indicate the formation of carboxylic acid groups at both ends and on the sidewalls of the CNT, responsible for mixing CNT with PANI[24]. As compared to pure PANI, PANI-CNT composite shows reduced in the ratio of benzoid to quinoid intensity indicating the stabilized form of nanocomposite and correlate the result of conductivity.

SEM images of MWCNT, functionalized MWCNT and PANI-CNT composite are shown in figure 3. PANI-CNT composite shows the homogeneous coating of PANI onto the CNT indicating that carbon nanotubes were well dispersed in polymer matrix. Rough surface and increased diameter of the composite indicated the coating of PANI over the CNTs since diameter of CNTs was 30-40 nm. Nanocomposite shows new interwoven fibrous structure acts as conductive pathway and leads to high conductivity as compared to that of pure PANI.

The electrical conductivity of PANI and PANI-CNT varies with temperature is shown in figure 4. PANI-CNT nanocomposite also follows the "thermal activated" behaviour as that of pure PANI. PANI-CNT interaction could facilitate the charge transfer process between them and influence the charge transport properties of composite. The room temperature conductivity of the nanocomposite along with transport parameters are reported in table 1. To find transport parameters of the polymer nanocomposites from the data of electrical conductivity, Ziller equation[24] has been used, which describes the interchain conductivity where only the neighbour variable range hoping $(\mathrm{VRH})$ of charge is considered.

$$
\sigma(T)=\sigma(o) \cdot e^{\left(-\frac{T o}{T}\right)^{1 / 2}}
$$

The plot of $\log \sigma(T)$ versus $T^{1 / 2}$ was found to be linear for pure PANI and PANI-CNT nanocomposite, hence To was determined from the slope of the line. 'To' the characteristic temperature, can be used to calculate the transport parame- ters such as charge localization length ( $\left.\alpha^{-1}\right)$, most probable hopping distance $(R)$ and charge hopping energy $(w)$ using the following relations,

$$
\begin{gathered}
\alpha^{-1}=\frac{8 N(E f) Z k}{T o} \\
R=\left(\frac{T o}{T}\right)^{1 / 2}\left(\frac{\alpha^{-1}}{4}\right) \\
w=Z k \frac{T o}{16}
\end{gathered}
$$

In these relations, $Z$ is the number of nearest neighbouring chains ( $\sim 4), k$ is Boltzmann constant and $N(E f)$ is the density of states per electron volt (2-ring unit suggested for polyaniline)[25]. MWCNT may serve "conducting bridge" connecting the PANI conducting domain. Thus functionalized MWCNT embedded in the PANI matrix have better conductivity with enhanced solubility and processability as compared to that of pure PANI.

Table 1. Transport parameters of PANI and PANI-CNT nanocomposites

\begin{tabular}{cccccc}
\hline Polymers & $\begin{array}{c}\sigma(\mathrm{S} / \mathrm{cm}) \text { at } \\
303 \mathrm{~K}\end{array}$ & $T o(\mathrm{~K})$ & $\alpha^{-1}(\mathrm{~nm})$ & $R(\mathrm{~nm})$ & $w(\mathrm{eV})$ \\
\hline PANI & 0.504 & 4085 & 2.22 & 2.04 & 0.088 \\
PANI-CNT & 1.954 & 4675 & 1.93 & 1.90 & 0.101 \\
\hline
\end{tabular}

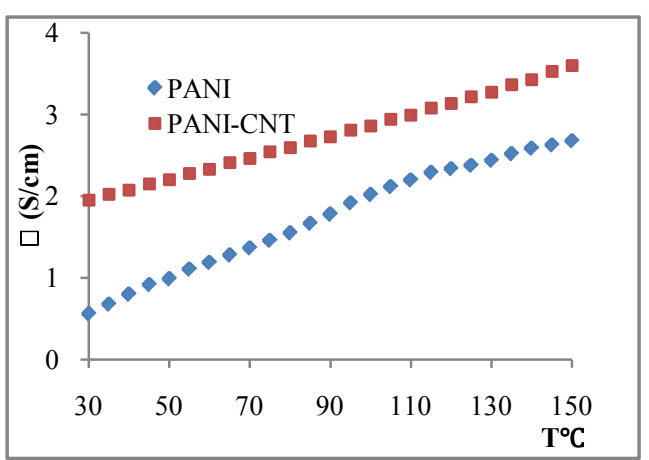

Figure 4. Electrical conductivity $(\sigma)$ of PANI and PANI-CNT composite

\section{Conclusions}

PANI-CNT nanocomposite was successfully synthesized by an in-situ chemical oxidative polymerization of aniline. FTIR, XRD and SEM show the effective structural modification and confirm the coating of PANI layer on the CNT surface. Incorporation of functionalized CNT into PANI matrix improved the transport properties of the nanocomposite. Transport parameters of PANI-CNT nanocomposite shows the composite is better electronic material than pure 
polyaniline.

\section{ACKNOWLEDGEMENTS}

The authors gratefully acknowledge UGC, New Delhi (India) for financial assistance provided to carry out this work through major research project file F No.39-540/2010 (SR).

\section{REFERENCES}

[1] Reghu Menon, Y. Cao, D. Moses, A. J. Heeger, "Counterion-induced precessibility of polyaniline: Transport at the metal-Insulator boundary", American Physical Society, Physical Review B, vol.47, no.4, pp.1758-1764, 1993.

[2] M. Ghosh, A. Barman, A. Das, A. K. Meikap, S. K. De, S. Chatterjee, "Electrical transport in paratoluene sulfonate doped polypyrrole films at low temperature", IEEE Xplore Digital Library, Journal of Applied Physics, vol. 83, no. 8, pp.4230-4235, 1998.

[3] A. Sarkar, P. Ghosh, A. K. Meikap, S. K. Chattopadhyay, S. K. Chatterjee, M. Ghosh, "Alternate and direct current conductivity of conducting polyaniline dispersed with poly vinyl alcohol and blended with methyl cellulose", Journal of Applied Physics, vol. 97, no.11, pp.113713-113716, 2005.

[4] P. Ghosh, A. Sarkar, A. K. Meikap, S. K. Chattopadhyay, S. K. Chatterjee, M. Ghosh, "Electron transport properties of cobalt doped polyaniline", IOP publishing, Journal of Physics D: Applied Physics, vol. 39, no. 14, pp. 3047-3050, 2006.

[5] P. M. Ajayan, O. Stephan, C. Colliex, D. Trauth, "Aligned Carbon Nanotube Arrays Formed by Cutting a Polymer Resin-Nanotube Composite", Science, vol. 265, no. 5176, pp. 1212-1214, 1994

[6] E. T. Thostensona, Z. Renb, T. W. Choua, "Advances in the science and technology of carbon nanotubes and their composites: a review", Composites Science and Technology, vol. 61, no. 13, pp. 1899-1912, 2001.

[7] P. J. F. Harris, "Carbon nanotubes composites", Maney for the Institute of Materials, Minerals and Mining and ASM publishing, International Materials Review, vol. 49, no.1, pp. 31-43, 2004.

[8] R. H. Baughman, A. A. Zakhidov, W. A. Heer, "Carbon nanotubes - the route toward applications", Science, vol. 297 no. 5582, pp.787-792, 2002.

[9] J. Sandler, M. S. P. Shaffer, T. Prasse, W. Bauhofer, K. Schulte, A. N. Windle, "Development of a dispersion process for carbon nanotubes in an epoxy matrix and the resulting electrical properties", Elsevier publishing, Polymer, vol. 40, pp. 5967-5971, 1999.

[10] A. B. Dalton, S. Collins, E. Munoz, J. M. Razal, V. H. Ebron, J. P. Ferraries, J. N. Coleman, B. G. Kim, R. H. Baughman, "Super-tough carbon-nanotube fibers", Nature, vol. 423, no. 6941, pp. 703-706, 2003.

[11] A. Modi, N. Koratkar, E. Lass, B. Wei, P. M. Ajayan, "Miniaturized gas ionization sensors using carbon nanotubes", Nature, vol. 424, no. 6945, pp.171-174, 2003.
[12] C. N. R. Rao, B. C. Satishkumar, A. Govindaraj, Manish Nath, "Nanotubes", Wiley publishing, Chem. Phys. Chem., vol. 2, no. 2, pp. 78-105, 2001.

[13] Sambhu Badra, Dipak Khastgir, Nikhil K. Singha, Joong Hee Lee, "Progress in preparation, processing and applications of polyaniline", Elsevier publishing, Progress in Polymer Science, vol. 34, pp. 783-810, 2009.

[14] H. Zengin, W. Zhou, J. Jin, R. Czerw, D. W. Smith, L. Echegoyen, D. L. Carroll, S. H. Foulger, J. Ballato, "Carbon nanotubes doped polyaniline", Wiley publishing, Advanced Materials, vol. 14, no. 20, pp. 1480-1483, 2002.

[15] E. Kymakis, G. A. J. Amaratunga, "Single-wall carbon nanotube/conjugated polymer photovoltaic devices", Applied Physics Letters, vol. 80, no. 1, pp. 112-114, 2002.

[16] H. S. Woo, R. Czerw, S. Webster, D. L. Carroll, "Organic light emitting diodes fabricated with single wall carbon nanotubes dispersed in a hole conducting buffer: the role of carbon nanotubes in a hole conducting polymer" Elsevier publishing, Synthetic Metals, vol. 116, no. 1, pp. 369-372, 2001.

[17] Yi Zhou, Zong-Yi Qin, Li Li, Yu Zhang, Yu-Ling Wei, Ling-Feng Wang, Mei-Fang Zhu, "Polyaniline/multi-walled carbon nanotube composites with core-shell structures as supercapacitor electrode materials", Elsevier publishing, Electrochimica Acta, vol. 55, pp. 3904-3908, 2010.

[18] Ravi Raj Vankayala, Wei-Jen Petrick Lai, Kuo-Chung Cheng, Kuo Chu Hwang, "Enhanced electrical conductivity of nylon 6 composite using polyaniline-coated multi-walled carbon nanotubes as additives", Elsevier publishing, Polymer, vol. 52, pp. 3337-3343, 2011.

[19] Qi Jianga, Guanggang Fu, Deyu Xie, Shengqiang Jiang, Zhiliang Chena, Bin Huang, Yong Zhao, "Preparation of carbon nanotube/polyaniline nanofiber by electrospinning", Elsevier publishing, Proceedia Engineering, vol. 27, pp. 72-76, 2012.

[20] Liang Ding, Qin Li, Dandan Zhou, Hao Cui, Hao An, Jianping Zhai, "Modification of glassy carbon electrode with polyaniline/multi-walled carbon nanotubes composite: Application of electro-reduction of bromate", Elsevier publishing, Journal of Electroanalytical Chemistry, vol. 668, pp. 44-50, 2012.

[21] Subodh Srivastava, S. S. Sharma, Sumit Kumar, Shweta Agrawal, M. Singh, Y. K. Vijay, "Characterization of gas sensing behaviour of multiwalled carbon nanotubes polyaniline composite films", Elsevier publishing, International Journal of Hydrogen Energy, vol. 34, pp. 8444-8450, 2009.

[22] S. B. Kondawar, S. R. Thakare, S. Bompilwar, V. Khati "Nanostructure Titania Reinforced Conducting Polymer Composites", World Scientific Publishing, International Journal of Modern Physics B, vol. 23, no. 15, pp. 3297-3304, 2009.

[23] S. H. S. Zein, L. C. Yeoh, S. P. Chai, A. R. Mohamed, "Synthesis of manganese oxide/carbon nanotube nanocomposites using wet chemical method", Elsevier publishing, Journal of Materials Processing Technology, vol. 190, no. 1-3, pp. 402-405, 2007.

[24] Tzong-Ming Wu, Yen-Wen Lin, "Doped polyaniline/multi-walled carbon nanotube composites: Preparation, characterization and properties", Elsevier publishing, Polymer, vol. 47, pp. 3576-3582, 2006. 

Nanocomposites Based on Carbon Nanotubes

D. C. Sindhimeshram, M. C. Gupta, "Transport proper- ties of substituted derivatives of poly(aniline)", Indian Journal of
Chemistry, vol. 34 A, no. 37, pp. 260-267, 1995. 